\title{
Theoretical research of needle-shaped harrow operation expended on soil pinching by needles
}

\author{
Alexander Serguntsov ${ }^{1, *}$, and Victoria Serguntsova ${ }^{2}$ \\ ${ }^{1}$ Kuban State Agrarian University named after I. T. Trubilin, 350044 Krasnodar, Russia \\ ${ }^{2}$ Adygeya State University, Maikop, Russia
}

\begin{abstract}
Needle-shaped harrows developed by us belong to harrows which represent known designs which are carrying out rolling in longitudinal-vertical plane. According to number of disks with needles, we have developed single- and double- disk harrows, under connection of which there can be formed a multi-disk harrow. Analysis shows that in order to improve impact of needles on soil in places of its pinching, it is necessary that in first disk with needles, the angle between which is equal to $\alpha$, and in other disk, which is located next to the first disk (i.e. parallel to first disk), needles are also at angle $\alpha$, however, these needles together with disk should be rotated in direction of movement of disks (or against direction of movement of these disks) at angle $\alpha / 2$. During the design of double-disk harrow, there was set the task of changing the design of needle harrow to ensure increase in uniformity of impact of needles on soil surface.
\end{abstract}

\section{Materials and methods of research}

Existing needle harrows are not used in operations of pre-emergence and post-emergence harrowing of grain crops, as their working bodies are not adapted to working conditions at shallow depths. Therefore, studies aimed at improving quality and energy performance of technological processes of soil treatment with needle harrows, angle of sharpening of which can vary, especially if they are used for shallow surface loosening of soils in climatic zones prone to wind erosion, are relevant. When designing needle harrows in order to minimize destructive force, it is necessary to ensure the depth of needle penetration into soil by the amount provided by processing technology. To do this, the design of harrow should assume its installation at given depth and achieve this depth during operation. In addition, material of needle should have sufficient strength and wear-resistance, so that initial (output) taper of needles is maintained during operation. [1].

\section{Results of research}

The efficiency of work spent to soil pinching by needles of needle-shaped harrow. The work spent to pinching soil with needle-shaped harrow can be defined as a result of number

\footnotetext{
* Corresponding author: sasha20089191@mail.ru
} 
of punctures applied to soil for work required to pinch one hole in soil. On Fig.1 there was presented a diagram of field's plot, which was sown with wide-row culture. Diagram shows three rows of plants, row spacing and protected bands [2]. Width of row spacing is designated as $B_{m}$, it consists of two protected bands in width $a_{p}$, where pinching cannot happen, and width of a part of row spacing, that is processed by pinching. Calculations are carried out under following assumptions: a) soil is homogeneous in its composition; b) all needles are lowered into soil at the same depth. Denote work spent on soil pinching with needles for certain period of time by $W$. This work will be presented as a result:

$$
W=W_{0} \cdot m_{g}
$$

where $W_{0}$ - work spent to implementation of one puncture;

$m_{g}-$ number of punctures made for given period of time.
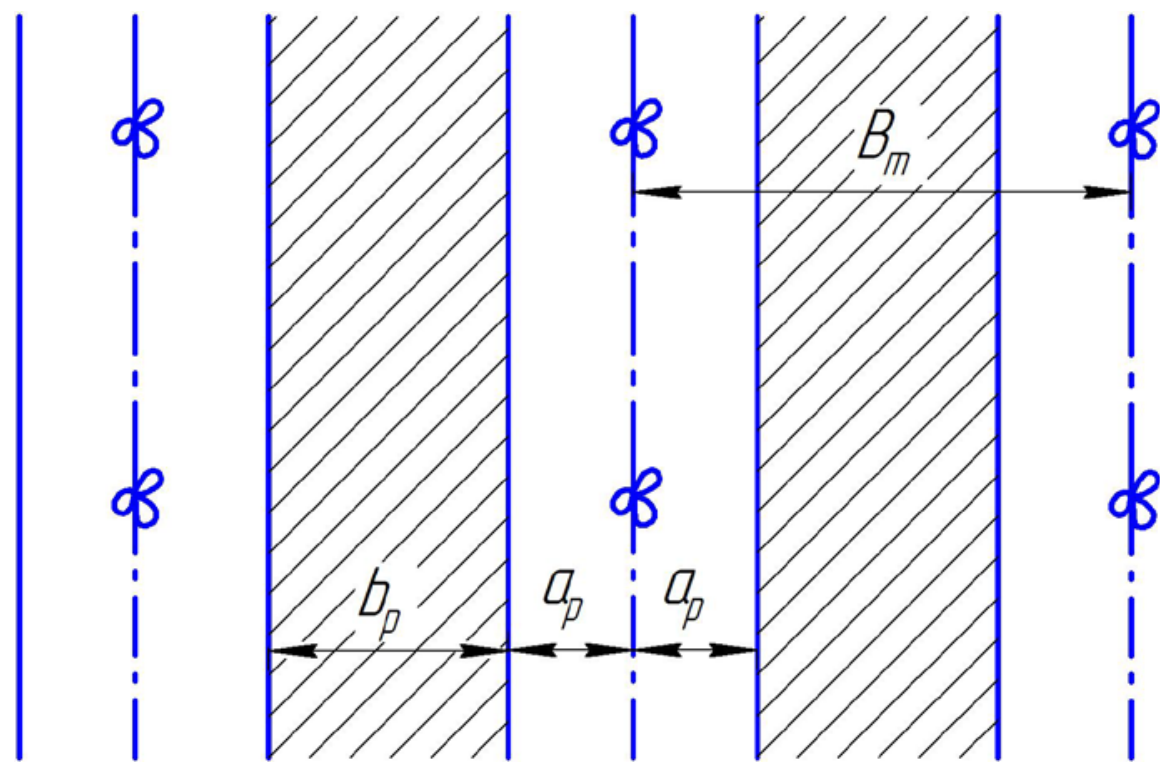

Fig. 1. Scheme of field area where wide-row crop is sown and where the soil is loosened in interval between rows (these areas are shaded): $a_{p}$ - protective band; $b_{p}$ - zone of harrow needles action, $B_{m}-$ width of row spacing.

Let us first determine value of $\mathrm{W}_{0}$. We must turn to Fig. 2, which shows graphical dependence of force $G$ of pressure of plunger of hardness tester on soil from depth $\lambda$ of its burial (soil crumpling diagram). In this diagram, $\lambda$ is depth of plunger into soil and $G$ is compression force of spring pressing plunger. The diagram is approximated by two straight lines $O A$ and $A B$, which characterize two phases of soil deformation. During first phase (line $O A$ ) soil resistance force increases linear deformation $\lambda$ proportionally. During first phase (line $O B$ ) the other phase is not changed in practice at increase of deformation $\lambda$ of soil resistance that is soil "flows" (changes in shape) under impact of constant pressure on it. 


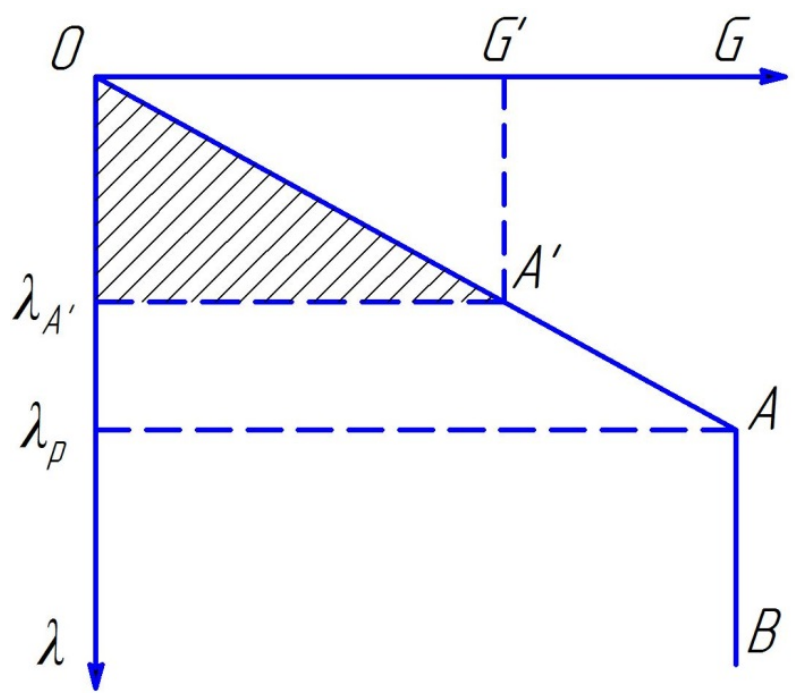

Fig. 2. Graphic dependence of depth $\lambda$ of needle penetration into soil on force $G$ of pressure on needle (diagram of soil crumpling)

We assume that punctures of soil are produced during the first phase, in which depth of penetration of plunger is small (i.e. smaller than distance $O \lambda_{p}$ on the diagram). Then strength $G$ at depth $\lambda$ divided by 2 , i.e., $G \lambda / 2$, so, for example, work on puncture by strength $G^{\prime}$ at depth of $O \lambda A^{\prime}$ to point $A^{\prime}$ (Fig. 2) equals $G^{\prime} \lambda A^{\prime} / 2$, i.e. equals the area of triangle $O A^{\prime} \lambda A^{\prime}$ at selected scale (shaded in Fig. 2).

This dependence is true when force $G$ and depth $\lambda$ from point $O$ to point $A$ in straight line, so that you can imagine dependence of work of $W_{0}$ on needle of one puncture from factors that affect as follows:

$$
W_{0}=\frac{G \cdot \lambda}{2}
$$

where $\lambda$-depth of puncture of soil by needle with strength $G$.

To determine the number of $m_{\mathrm{g}}$, we take into account that number of $n_{\mathrm{g}}$ punctures is applied per one turn of needle harrow disk (in our case it is 16). The number of $n_{\mathrm{g}}$ punctures that a section performs per a turn is $n_{\mathrm{g}} m_{\mathrm{d}}$, where $m_{\mathrm{d}}$ is number of disks in section (in our case $m_{\mathrm{d}}=2$ ) [4].

Number of disk's turns on run with length $L_{\mathrm{g}}$ equals $L_{\mathrm{g}} / L_{\mathrm{k}}$, where $L_{\mathrm{k}}-$ length of one disk's contour, it is defined by the formula (3), if angle $\alpha$ stands in it. So,

$$
\frac{L_{g}}{L_{k}}=\frac{L_{g}}{2 n_{g} \cdot r_{d g} \cdot \sin \frac{\pi}{n_{g}}}
$$

The number of $m_{\mathrm{g}}$ punctures which section will make for whole period of time will be as follows.

$$
m_{g}=n_{g} \cdot m_{d} \cdot \frac{L_{g}}{2 n_{g} \cdot r_{d g} \cdot \sin \frac{\pi}{n_{g}}}=\frac{L_{g} \cdot m_{d}}{2 r_{d g} \cdot \sin \frac{\pi}{n_{g}}}
$$

Substituting value $W_{0}$ in (2) and $m_{\mathrm{g}}$ in (4) in the equality (1), we find $W$ : 


$$
W=\frac{G \lambda}{2}=\frac{L_{g} \cdot m_{d}}{2 r_{d g} \cdot \sin \frac{\pi}{n_{g}}}=\frac{G \cdot \lambda \cdot L_{g} \cdot m_{d}}{4 r_{d g} \cdot \sin \frac{\pi}{n_{g}}}
$$

So the work expended on soil punching by needle harrow on run with length $\mathrm{L}_{\mathrm{g}}$ is calculated. We can see in the formula that work $W$ is much than number of disks, number of needles on disk, pressure force of needles to soil and depth $\lambda$ of a puncture [5]. If length ofrun $\mathrm{L}_{\mathrm{g}}$ multiplies width of row space $\mathrm{B}_{\mathrm{m}}$, it equals $\mathrm{b}_{\mathrm{p}}+2 \mathrm{a}_{\mathrm{p}}$, we will obtain the processed area $F_{\mathrm{p}}$ :

$$
F_{p}=B_{m} \cdot L_{g}=L_{g} \cdot\left(b_{p}+2 a_{p}\right)
$$

Area processed for one second, $F_{\mathrm{c}}$ will equal:

$$
F_{c}=\frac{F_{p}}{t}=\frac{L_{g} \cdot\left(b_{p}+2 a_{p}\right)}{t}
$$

where $t$ - unit operating time, s.

If work of $W$ in $\mathrm{J}$ is divided by time $t$ of needle harrow operation during movement in run in $s$, then we get power consumption $N_{m}$.

Time $t$ equals $L_{\mathrm{g}} / v_{m}$, where $v_{m}-$ unit velocity movement.

Then

$$
N_{m}=\frac{G \cdot m_{d} \cdot \lambda \cdot v_{m}}{4 r_{d g} \cdot \sin \frac{\pi}{n_{g}}}
$$

\section{Conclusions}

The work required to pinch soil with needles of a harrow increases with number of needle disks in a machine [6], force of needle pressure on soil, depth of needle penetration into soil and length of run, and with decrease in radius of a disk and angle between needles in a disk. Power that is consumed by needle harrow increases with increasing velocity of machine, number of disks with needles, pressure force of needles on soil and decrease in radius of a disk.

\section{References}

1. Khomenko M.S., Prospects of use of soil-processing machines with passive and active bodies. // Mechanization and electrification of agriculture. 1987. - №5. - p.26-28.

2. Williams V.R. Soil science, agriculture with main basics of soil science. $-5^{\text {th }}$ of edition. - M., 1947. - 456 p.

3. Goryachkin V.P. Collected works: in 3 volumes. - M.: Kolos, 1968. - Volume III. - 384

p.

4. Belyaev N.M. Soil-processing machines with active working bodies. // Mechanization and electrification of agriculture. 1978. - №2. - p.54-57.

5. Vasilenko I.F. Experimental theory of cutting units of harvesters. Theory, construction and production of agricultural machines. Under edition V.P. Goryachkin. Volume IV. M. L.: Selkhozgiz, 1936. - 583 p.

6. Unit for soil processing with introduction of fertilizers / Patent for invention RUS 2629265. Published 28.08.2017. Authors: Maslov G.G., Serguntsov A.S. 